\title{
Article
}

\section{On the Shape of Nucleons at High Energies}

\author{
Maksim L. Nekrasov (D)
}

Institute for High Energy Physics, NRC “Kurchatov Institute”, Protvino 142281, Russia; nekrasov@ihep.ru

\begin{abstract}
A scenario of the evolution of the shape of nucleons with increasing energy is described in the framework of an extended parton model, which consistently takes into account the transverse motion of the partons. At the energy $E$ up to LHC, the nucleons have the form of a spheroid which expands as $\sqrt{\ln E}$ in the transverse directions and grows linearly in $E$ in the longitudinal direction. With a further increase in the energy, a mode of correlated behavior of the partons is established, which stops the longitudinal growth. Simultaneously, the expansion in the transverse directions changes to $\ln E$, and a hollow mostly free of partons is formed inside the nucleons along the central axis in the direction of their motion. Numerical estimates of the corresponding parameters are obtained.
\end{abstract}

Keywords: proton shape; interaction region; impact parameter; cross section; diffraction cone; parton model

\section{Introduction}

The spatial shape of hadrons is one of the intriguing problems in the hadron physics. A closely related problem is the spatial region of interaction between the hadrons in elastic collisions at high energies. An independent solution to these problems would explain the behavior of many characteristics of hadron scattering. Accordingly, the study of these problems has been at the forefront of theoretical research for many years [1-12].

Attention is usually mainly paid to the transverse sizes of hadrons and the region of their Shape of Nucleons at High Energies. Particles 2021, 4, 381-390. https:// doi.org/10.3390/particles4030032

Academic Editor: Kazuharu Bamba

Received: 14 July 2021

Accepted: 18 August 2021

Published: 21 August 2021

Publisher's Note: MDPI stays neutral with regard to jurisdictional claims in published maps and institutional affiliations. interaction. In the first approximation, they can be judged by the total cross section. However, more accurate information is provided by the slope parameter $B$ of the diffraction cone in the elastic collisions. Namely, due to relation $B=\left\langle b^{2}\right\rangle / 2$, which links $B$ with the mean square of the impact parameter $b$, one can judge the transverse sizes of the interaction region. The transverse sizes of the hadrons must be related to $\left\langle b^{2}\right\rangle$, as well. Experimental data on the nucleon scattering indicate a growth of $B(s)$ as $\ln s$ up to the Tevatron energy [13], and then an accelerated growth at the LHC energies [14], possibly as $\ln ^{2} s$ [15], see Figure 1. The total and elastic cross-sections exhibit similar behavior [14].

In theory, such behavior is predicted by the Regge model. Really, at moderately high energies it describes the elastic scattering of hadrons via the one-pomeron exchange. This leads to $B=2 \alpha_{0}^{\prime} \ln \left(s / s_{0}\right)$, where $\alpha_{0}^{\prime}$ is the slope of the pomeron trajectory. At ultra-high energies, the multi-pomeron exchanges lead to $B \propto \ln ^{2} s$ and the same behavior for the total cross-section. The transition to the multi-pomeron exchange mode is expected in the 2-7 TeV region [15].

This behavior must be explained at the "microscopic" level of quarks and gluons, as well. Unfortunately, the means of perturbative QCD can hardly be useful in this case since the relevant processes occur at extremely small transfers. However, as they occur at high energies, the parton model is suitable for appropriate consideration. In that way, the result, which became classical, was obtained about the "diffusion" growth of the transverse sizes of hadrons as $\sqrt{\ln s}$ [6], which means the behavior $B(s) \propto \ln s$, observed at the moderately high energies. In the analysis [6], hadrons were considered free-moving, which can be associated with their elastic scattering in the limit $t=0$ (excluding electromagnetic interactions). Recently an extension of this approach was proposed [12], which took into 
account in more detail the transverse motion of the partons. The latter analysis predicted a faster growth of the transverse sizes of hadrons in the transition to the ultra-high energies, from $\sqrt{\ln s}$ to $\ln s$. This means the transition of the behavior of $B(s)$ from $\ln s$ to $\ln ^{2} s$.

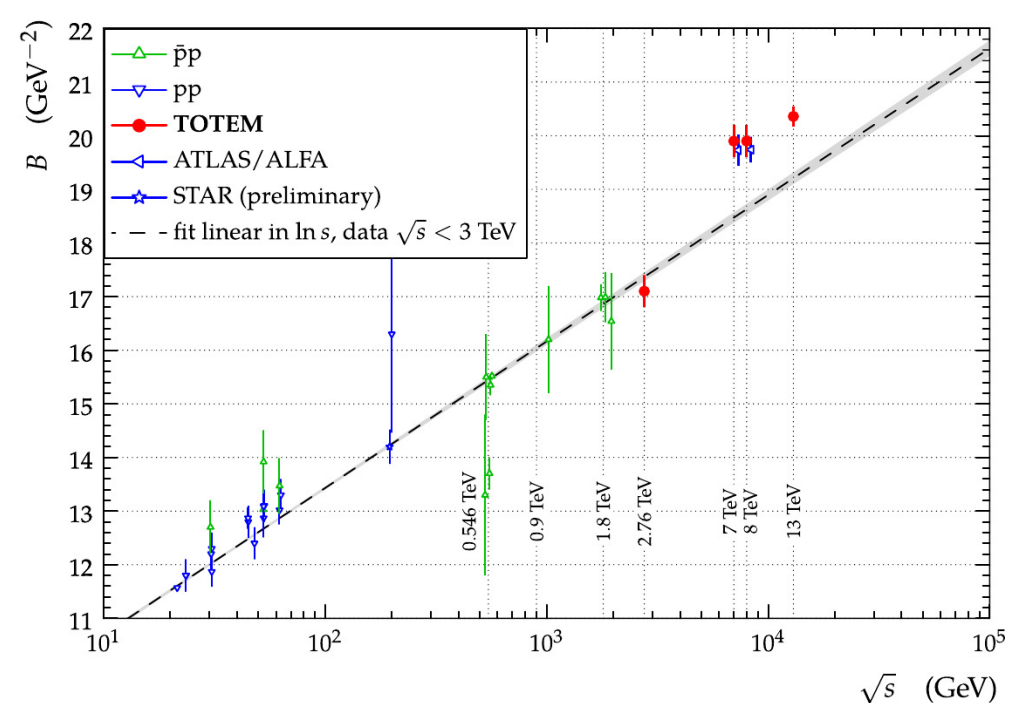

Figure 1. The energy dependence of the slope B of the diffraction cone [14].

Unfortunately, the situation with the longitudinal sizes of hadrons remains uncertain. The point is that no experimental criteria for estimating the longitudinal hadron sizes have been proposed so far. Therefore, the reasoning on this topic is rather speculative. Actually, two points of view currently dominate. The first one is associated with the concept of a hadron at rest as a rigid ball or bag of approximately spherical shape. Based on this, the fast hadron due to Lorentz contraction should look like a highly flattened pancake with a longitudinal size of the order of $1 / E$, where $E$ is the energy of the hadron. According to the other point of view, [6] the longitudinal sizes are determined by the minimum momenta of the partons, at which they still belong to the hadron (i.e. separated from the vacuum). From the latter point of view, the longitudinal size of the hadron does not depend on the energy and is of the order of $1 / \mu$, where $\mu$ is the soft scale in the nucleon.

However, there is a third point of view, which assumes that hadrons take a certain shape under certain conditions of interaction. Following this idea and taking into account the short-range nature of strong interactions, the shape of hadrons and the region of their interaction are mutually determined. In particular, the transverse sizes of hadrons must be consistent with the transverse sizes of the interaction region, and vice versa. Similarly one should approach the question of the longitudinal sizes. Currently, it is widely believed that the longitudinal sizes of the hadron interaction region grow linearly with the energy [2-7]. So, if this is the case, then one should assume that the longitudinal sizes of fast hadrons grow linearly with the energy, as well.

In fact, however, the latter assumption seems paradoxical, since starting at certain energies the longitudinal sizes should reach the macroscopic values, which is difficult to accept. As far as we know, this issue has not been discussed anywhere. Apparently, by default it was assumed that upon reaching certain energies further growth should be stopped. However, no ideas were proposed for the implementation of this scenario.

In this article, we develop an idea that the reason for limiting the longitudinal growth of hadrons is the same that leads to the accelerated expansion in the transverse directions at ultra-high energies. The underlying reason is the emergence of the correlations between the partons at ultra-high energies, which ultimately is related to the confinement phenomenon. Moreover, for the same reason a hollow appears inside the hadrons along the central axis in the direction of their motion. We make estimates of the corresponding parameters in the case of nucleons. In this regard, below we refer to nucleons, although the qualitative results can be applied to other hadrons, as well. 
The structure of the paper is as follows. In Section 2, we give necessary definitions and describe the evolution of the shape of nucleons in the transverse projection. Section 3 considers the evolution in the longitudinal direction. The results are discussed in Section 4.

\section{Fast Nucleons in the Transverse Projection}

We proceed from the idea that nucleon at rest can be represented in the first approximation as a bound state of three light quarks. Of course, the quarks can virtually split into other quarks and gluons, but they immediately recombine back, so that the average number of quarks in the nucleon at rest is approximately three. This can be understood as follows. Each initial quark in the nucleon is distributed with a variance that can be estimated as the radius $R_{\mathrm{v}}$ of the nucleon at rest. In turn, if quarks are distributed in the configuration space, they are distributed in the momentum space, as well, with a variance of $\mu \simeq 1 / R_{\mathrm{v}}$, which is roughly $0.3 \mathrm{GeV}$. Furthermore, since the light quarks are almost massless, their average energy and average momenta squared are connected via the relation $\left\langle E^{2}\right\rangle \simeq\left\langle p^{2}\right\rangle$. However, the average quark momentum is zero. So, $\left\langle p^{2}\right\rangle$ is essentially the momentum variance. Consequently, $\left\langle E^{2}\right\rangle \simeq \mu^{2}$ and $\mu$ can be interpreted as the effective quark mass in the nucleon. Owing to its large value, the splitting of quarks in the nucleon at rest is strongly suppressed.

A completely different picture occurs in a fast-moving nucleon. Because of the excess energy, the fast quarks and gluons-hereinafter referred to as partons-split freely, acquiring effective ("transverse") mass due to their transverse motion. Simultaneously, they lose the longitudinal momentum and the energy, until they become slow. When they become slow, they can no longer split due to their large effective mass, and the development of the decay cascade is completed.

In the above processes, an important feature is that each splitting is accompanied by a shift in the position and transverse momentum of newly formed parton relative to the parton-parent. In the configuration space, this leads to the shift of the partons during splitting to the periphery. With increasing energy of the nucleon the decay chain becomes longer, and the last partons in the cascades become more and more distant from the geometric center. Correspondingly, the transverse size of the nucleon increases with the energy. In the case of uncorrelated partons motion, the increase is proportional to $\sqrt{\ln s}$ (the "diffusion motion") [6] and in the case of correlated motion it may be proportional to ln s [1]. The latter option was confirmed in [12]. Moreover, [12] showed that in the former case the transverse root-mean-square (RMS) momenta of the last partons in the cascades also grow as $\sqrt{\ln s}$, but in the latter case, they are limited.

Let us recall the basic definitions from [12]. We define the effective transverse radius $\mathcal{R}(s)$ of the nucleon as the average variance of the distance between the peripheral partons and the central axis in the direction of motion of the nucleon. Peripheral partons are those that arise at the end of the cascades, and they have the greatest variance among the partons in cascades. It should be emphasized that the number of patrons in the cascades is not fixed (the cascades may be incomplete) and is determined by a certain probability distribution. The above-mentioned average of the variance is determined by this probability distribution. Similarly, we define the size $\mathcal{K}(s)$ of the nucleon in the transverse momentum space as the average variance of the transverse momenta of the peripheral partons. The latter partons also have the largest RMS transverse momenta relative to the geometric center of the nucleon.

\subsection{The Mode of Uncorrelated Motion of the Partons}

One of fundamental results of [12] is the observation that in the mode of uncorrelated ("diffusive") motion of the partons both $\mathcal{R}$ and $\mathcal{K}$ are proportional to the average number $\bar{N}$ of partons in cascades,

$$
\begin{gathered}
\mathcal{R}^{2}=\bar{N} \mu^{-2}, \\
\mathcal{K}^{2}=\bar{N} \mu^{2} .
\end{gathered}
$$


Here $\mu$ is the variance of the transverse parton momentum relative to the parent parton. This quantity is independent of the longitudinal momentum of the nucleon, and coincides with that in the nucleon at rest (the latter is obvious when $\bar{N}=1$ ). Let us emphasize once again, that (1) and (2) include average values in the cascades. However, the same relations are valid for the corresponding maximum values, $N_{m}, \mathcal{R}_{m}^{2}$, and $\mathcal{K}_{m}^{2}$, related to the fully developed cascades.

Two important consequences may be immediately deduced from (1) and (2). The first one is that the behavior of $\mathcal{R}^{2}$ and $\mathcal{K}^{2}$ is fully determined by the average number of the partons $\bar{N}$. In the parton model $\bar{N}$ increases asymptotically as $\ln s[1,6]$. So $\mathcal{R}^{2}$ and $\mathcal{K}^{2}$ should increase with the energy as $\ln s$. The second consequence is that the average parton density $\varrho$ in the transverse projection is a constant, independent of the nucleon energy,

$$
\varrho \equiv 3 \bar{N} / S=3 \mu^{2} / \pi
$$

Here $S=\pi \mathcal{R}^{2}$, and 3 is the number of the cascades in a free-moving nucleon. Actually, the number of cascades is determined by the number of valence quarks, if the development of cascades goes without branches. The latter property is confirmed at high energies in the leading approximation, see e.g., [1]. At low energies, this is our assumption. (If branching nevertheless occurs at low energies, then 3 should be replaced by another larger number, which should no longer change with increasing energy at high energies). The fact that $\varrho$ is constant means that nucleons can expand unrestrictedly with increasing the energy as new partons are formed, without any contradiction to the confinement.

Parameter $\mu$ must be determined more precisely. The problem is that the sizes of the nucleons in strong and electromagnetic interactions differ from each other. Recently [11] defined the transverse radius of strong-interacting valence quark core of a nucleon as $\mathcal{R}_{\mathrm{v}}^{2}=\left\langle b_{0}^{2}\right\rangle / 2$, where $\left\langle b_{0}^{2}\right\rangle$ is the impact parameter at $s=s_{0}$, and $s_{0}$ is the energy at which the colliding nucleons cease to overlap with each other in the impact parameter plane. Phenomenologically, $s_{0}$ is the energy at which the growth rate of $B(s)$ changes from very fast to logarithmic. So at $s \geq s_{0}$ (but not too large $s$ ) the slope parameter is

$$
B(s)=B_{0}+2 \alpha_{0}^{\prime} \ln \left(s / s_{0}\right),
$$

where $\alpha_{0}^{\prime}$ is a phenomenological parameter. The estimates of [11] are as follows: $\sqrt{s_{0}} \approx$ $10 \mathrm{GeV}, B_{0} \equiv B\left(s_{0}\right)=11.10 \pm 0.26 \mathrm{GeV}^{-2}$, and in view of $B_{0}=\left\langle b_{0}^{2}\right\rangle / 2, \mathcal{R}_{\mathrm{v}}=\sqrt{B_{0}}=$ $0.656 \pm 0.008 \mathrm{fm}$. Notice that in this case the three-dimensional radius of the valence quark core is $R_{\mathrm{v}}^{2}=(3 / 2) \mathcal{R}_{\mathrm{v}}^{2}=(0.805 \pm 0.009 \mathrm{fm})^{2}$.

We generalize the above approach by supplementing it with the assumption that for any $s \geq s_{0}$ the actual transverse size of a nucleon is determined by the relation $\mathcal{R}^{2}=\left\langle b^{2}\right\rangle / 2$. So, given $\left\langle b^{2}\right\rangle / 2=B$, we have

$$
\mathcal{R}^{2}(s)=\mathcal{R}_{0}^{2}+2 \alpha_{0}^{\prime} \ln \left(s / s_{0}\right),
$$

where $\mathcal{R}_{0}^{2}=B_{0}$. In fact, (5) is a realization of the concept that the region of interaction between nucleons must be comparable with their actual sizes. Reversing the logic, we can say that with increasing the energy the radii of nucleons increase and, therefore, the RMS impact parameter increases.

It should be emphasized that in our approach $\mathcal{R}_{\mathrm{v}}$ differs from $\mathcal{R}_{0}$. To get $\mathcal{R}_{\mathrm{v}}$, we must go down in energy to the nucleon at rest, i.e. to $\sqrt{s} / 2=M$ where $M$ is the nucleon mass. The basis for this extrapolation is that relation (5) is a consequence of the diffusion process which starts from the rest state of the nucleon. (In contrast, Formula (4) is inapplicable at $s<s_{0}$, since at these energies nucleons are scattering in the overlap mode of their physical sizes.) Taking this into account, we get

$$
\mathcal{R}_{\mathrm{v}}^{2}=\mathcal{R}_{0}^{2}+2 \alpha_{0}^{\prime} \ln \left(4 M^{2} / s_{0}\right) .
$$


With $\alpha_{0}^{\prime}=0.25 \mathrm{GeV}^{-2}$ [13], this leads to $\mathcal{R}_{\mathrm{v}}=0.60 \mathrm{fm}\left(R_{\mathrm{v}}=0.74 \mathrm{fm}\right)$. Hence we have $\mu=1 / \mathcal{R}_{\mathrm{v}}=0.33 \mathrm{GeV}$ and $\varrho=(0.62 \mathrm{fm})^{-2}$. Note that $\mathcal{R}_{\mathrm{v}}$ is weakly dependent on $\alpha_{0}^{\prime}$. For example, $\alpha_{0}^{\prime}=0.321 \mathrm{GeV}^{-2}$ [16] gives $\mathcal{R}_{\mathrm{v}}=0.59 \mathrm{fm}$ and, within the accepted accuracy, the same value for $\mu$. Below, we do estimates with $\alpha_{0}^{\prime}=0.25 \mathrm{GeV}^{-2}$ and $\mu=0.33 \mathrm{GeV}$.

Now we turn to other formulas. In view of (1) and (5) we have

$$
\bar{N}=\mu^{2}\left[\mathcal{R}_{0}^{2}+2 \alpha_{0}^{\prime} \ln \left(s / s_{0}\right)\right] .
$$

This gives $\bar{N}_{\mathrm{v}}=1$ at $\sqrt{s}=2 M$, as required. An important characteristic is the maximum number $N_{m}$ of partons in cascades. At high energies in the multi-peripheral picture $N_{m}=$ $\gamma \ln (2 P / \mu)$, where $\gamma \simeq 1 / \ln 2$ and $P$ is the longitudinal momentum of the cascade [1,6]. In the case of nucleon at rest, $P$ makes sense of the RMS momentum of the valence quarks ( $P=\mu$ leads to $\left.N_{m}=1\right)$. In the case of a fast nucleon, assuming its momentum is equally distributed between the cascades, we have $P=\sqrt{s} / 6$. This gives

$$
N_{m} \simeq \gamma \ln \frac{\sqrt{s}}{3 \mu} .
$$

(This approximation also works well for the nucleon at rest, since $\sqrt{s}=2 M$ gives $N_{m} \simeq$ 0.94, which is close to 1.) Based on (7) and (8), we define the parameter of saturation of the cascades, $\varkappa=\bar{N} / N_{m}$. It monotonically decreases from 1 at $\sqrt{s}=2 M$ to $4 \alpha_{0}^{\prime} \mu^{2} / \gamma \simeq 0.07$ at $s \rightarrow \infty$.

Finally, in view of (2), (6), and (7), the RMS transverse momentum of the peripheral parton is

$$
\mathcal{K}^{2}(s)=\mu^{2}\left[1+2 \alpha_{0}^{\prime} \mu^{2} \ln \left(s / 4 M^{2}\right)\right] .
$$

Obviously, the growth of $\mathcal{K}^{2}$ with $s$ should not continue indefinitely, since too large transverse motions would lead to problems with integrity of the nucleon. So, the "diffusive" mode should end at some energies. It is worth noting that the maximum momentum in the cascades $\mathcal{K}_{m}$ is more critical in this sense, since the growth of $\mathcal{K}_{m}$ exceeds that of $\mathcal{K}$ in view of $\mathcal{K}_{m}=\mathcal{K} / \sqrt{\varkappa}$.

Table 1 shows the values of the above quantities for various $s$, starting from $s=s_{0}$ and further including the energies of ISR, Sp $\bar{p} S$, Tevatron, and LHC. For illustration, we also show results for $\sqrt{s}=100 \mathrm{TeV}$. We note that $\mathcal{K}$ gives the lower estimate for the average transverse momenta of secondary particles in the inelastic processes. The mentioned averages are as follows: $0.4 \mathrm{GeV}$ at $\sqrt{s}=31.5-63 \mathrm{GeV}$ [17], $0.42 \mathrm{GeV}$ at $\sqrt{s}=540 \mathrm{GeV}$ [18], $0.50 \mathrm{GeV}$ at $2.36 \mathrm{TeV}$ [19], $0.55 \mathrm{GeV}$ at $7 \mathrm{TeV}$ [20]. So, the limited growth of $\mathcal{K}$ with the energy is quite consistent with the data.

Table 1. Estimated parameters in the mode of uncorrelated motion of the partons: the effective transverse radius of the nucleon $\mathcal{R}$; transverse RMS momenta of the peripheral partons, average $\mathcal{K}$ and maximum $\mathcal{K}_{m}$ in cascades; the number of the partons in cascades, average $\bar{N}$ and maximum $N_{m}$; cascade saturation parameter $\varkappa$.

\begin{tabular}{lllllll}
\hline$\sqrt{s}[\mathrm{GeV}]$ & $\mathcal{R}[\mathrm{fm}]$ & $\mathcal{K}[\mathrm{GeV}]$ & $\mathcal{K}_{m}[\mathrm{GeV}]$ & $\bar{N}$ & $\boldsymbol{N}_{m}$ & $\varkappa$ \\
\hline 10 & 0.66 & 0.35 & 0.60 & 1.18 & 3.36 & 0.35 \\
50 & 0.70 & 0.38 & 0.78 & 1.35 & 5.68 & 0.24 \\
500 & 0.76 & 0.41 & 0.98 & 1.59 & 9.00 & 0.18 \\
2000 & 0.80 & 0.43 & 1.08 & 1.74 & 11.00 & 0.16 \\
7000 & 0.83 & 0.45 & 1.17 & 1.87 & 12.81 & 0.15 \\
13,000 & 0.84 & 0.45 & 1.21 & 1.94 & 13.70 & 0.14 \\
100,000 & 0.89 & 0.48 & 1.33 & 2.15 & 16.64 & 0.13 \\
\hline
\end{tabular}




\subsection{The mode of Correlated Motion of the Partons}

The fundamental feature in the mode of correlated motion is a fixed value of $\mathcal{K}$ and, at the same time, an accelerated growth of $\mathcal{R}$, and in such a way that the uncertainty relation for cascade processes is satisfied [12]. In the notation of this work, the latter relation is

$$
\mathcal{R} \mathcal{K} \geq \bar{N} .
$$

(Recall that limiting the RMS momentum does not prohibit its large fluctuations, but means they have low probability). So, if for $s=s_{1}$ the grows of $\mathcal{K}$ stops at a certain $\mathcal{K}=\mathcal{K}_{1}$, then $\mathcal{R}$ continues evolution for $s>s_{1}$ in accordance with the relation

$$
\mathcal{R} \geq \bar{N} / \mathcal{K}_{1}
$$

Let us now take into account that $B(s) \sim \ln ^{2} s$ at ultra-high $s$. Assuming that the change in the behavior of $B(s)$ occurs at $s=s_{1}$, we get for $s \geq s_{1}$

$$
\widehat{B}(s)=B_{1}+A \ln ^{2}\left(s / s_{1}\right) .
$$

Here $A$ is a parameter, $B_{1}=B\left(s_{1}\right)$ provides crosslinking with (4) at $s=s_{1}$. Symbol " $\leadsto$ " at the top means that the corresponding quantity relates to the mode $s \geq s_{1}$.

Next, we apply the provision that the size of strongly interacting nucleons is half the impact parameter in quadrature. Given $\left\langle\widehat{b}^{2}\right\rangle / 2=\widehat{B}$, this leads to

$$
\widehat{\mathcal{R}}^{2}(s)=\mathcal{R}_{1}^{2}+A \ln ^{2}\left(s / s_{1}\right),
$$

where $\mathcal{R}_{1}^{2}=B_{1}$. In view of (11) and (13) we also have

$$
\widehat{\bar{N}} \simeq \mathcal{K}_{1}\left[\mathcal{R}_{1}^{2}+A \ln ^{2}\left(s / s_{1}\right)\right]^{1 / 2}
$$

This means $\widehat{\widehat{N}} \sim \ln s$ as $s \rightarrow \infty$. The crosslinking between (14) and (7) at $s=s_{1}$ is automatic if (10) implies equality. However, the equality is not necessary if the mode change occurs in a finite region.

The consequence of (13) and (14) is a fundamentally different behavior of the average parton density in the transverse projection,

$$
\widehat{\varrho}=\left(3 \mathcal{K}_{1} / \pi\right)\left[\mathcal{R}_{1}^{2}+A \ln ^{2}\left(s / s_{1}\right)\right]^{-1 / 2} .
$$

Recall that in the uncorrelated ("diffusion") mode $\varrho$ is an energy-independent constant. Unlike that, $\widehat{\varrho}$ decreases with increasing the energy. This implies that each parton occupies more and more space in the impact parameter plane and, therefore, the distance between the partons increases indefinitely. Obviously, this is incompatible with the confinement. The only reasonable way to resolve the contradiction is to assume the appearance of an expanding hollow inside the nucleons, mostly free of partons. Due to the symmetry, the hollow is located along the geometric central axis of the nucleon. So, the nucleon in a rough approximation takes the form of a ring in the transverse projection.

The hollow starts forming when the density reaches a critical (small) value. Formula (15) does not work in the presence of the hollow, but we can express its radius $r$ through the local density $\widehat{\varrho}$. Based on the definition $\widehat{\varrho}=3 \widehat{\bar{N}} / S, S=\left[\pi\left(\widehat{\mathcal{R}}^{2}-r^{2}\right)\right]$, we get

$$
\widehat{r}^{2}=\widehat{\mathcal{R}}\left(\widehat{\mathcal{R}}-v \mathcal{R}_{1}\right),
$$

where $v=\varrho / \widehat{\varrho} \geq 1$. In the limiting case $v=1$, the hollow starts to form at $s=s_{1}$. In the general case, the hollow appears when the outer radius $\widehat{\mathcal{R}}$ of the nucleon reaches $v \mathcal{R}_{1}$. The thickness of the ring at this moment is maximum (equals the outer radius), and then it 
monotonically decreases to $v \mathcal{R}_{1} / 2$ with increasing $s$. In any case $r$ grows asymptotically as $\ln s$, following $\widehat{\mathcal{R}}$ minus the thickness of the ring.

Table 2 shows numerical estimates in the case $v=1$ at $\sqrt{s_{1}}=2 \mathrm{TeV}$ and $\sqrt{s_{1}}=7 \mathrm{TeV}$ (the results are separated by "/"). Based on the fit [15], we use $A=0.029$.

In conclusion, we note that it is a priori unclear whether the hollow inside the nucleons affects the region of their interaction. However, the idea of the appearance at high energies of a hollow in the nucleon interaction region is intensively discussed, see [21-23] and the references therein. Irrespective of this, recent analysis [24] of the TOTEM data at $13 \mathrm{TeV}$ led to the conclusion about the multilayer structure of the protons, with the spatial size of the inner layer near $0.45 \mathrm{fm}$. This value should be compared with $r$ in Table 2.

Table 2. Estimated parameters in the mode of correlated motion at $\sqrt{s_{1}}=2 \mathrm{TeV} / 7 \mathrm{TeV}$. The notation is similar to that of Table 1.

\begin{tabular}{llllll}
\hline$\sqrt{s}[\mathrm{GeV}]$ & $\widehat{\mathcal{R}}[\mathrm{fm}]$ & $r[\mathrm{fm}]$ & $\widehat{\hat{N}}$ & $N_{m}$ & $\widehat{\varkappa}$ \\
\hline 2000 & 0.80 & 0. & 1.74 & 11.00 & 0.16 \\
7000 & $0.90 / 0.83$ & $0.31 / 0$. & $1.97 / 1.87$ & 12.81 & $0.15 / 0.15$ \\
13,000 & $1.02 / 0.85$ & $0.48 / 0.15$ & $2.23 / 1.93$ & 13.70 & $0.16 / 0.14$ \\
100,000 & $1.55 / 1.23$ & $1.08 / 0.70$ & $3.39 / 2.78$ & 16.64 & $0.20 / 0.17$ \\
\hline
\end{tabular}

\section{The Longitudinal Size}

Above, we have identified two features that indicate a change in the mode of motion of the partons. One of them is phenomenological (the change in the behavior of the slope of the diffraction cone), and the other is the internal inconsistency (unrestricted growth of the RMS transverse momenta in the mode of uncorrelated motion). However, the fundamental reason for the mode change remains unknown. We will see below that it is related to the longitudinal size of nucleons.

We mentioned in the Introduction the widespread opinion about the longitudinal region of interaction of fast nucleons, that it increases linearly with increasing energy of the collision [2-7]. From the viewpoint of the parton model this can be explained by the fact that nucleons interact in collisions mainly through slow partons, and the time of development of quantum fluctuations leading to slow partons is proportional to the energy of the nucleons $[1,6]$. For this reason the variance of the hadron interaction region in the longitudinal direction increases linearly with the energy. Now we recall that the interaction region of slow partons is of the order of $1 / \mu$. Hence it follows that the longitudinal sizes of nucleons and of their interaction region must coincide with the $1 / \mu$-accuracy. Based on this, we conclude that the longitudinal size of the nucleons increases linearly with their energy.

The next question is how far the longitudinal size can grow. To answer, we relate the number of partons in the rapidly moving nucleon to its three-dimensional volume. We have seen above that the number of partons increases logarithmically with the energy, the cross-section area also increases logarithmically (in the mode of uncorrelated motion of partons), and the longitudinal size of the nucleon increases, too. This means that the three-dimensional density of the partons decreases with increasing energy, i.e. partons become less densely packed in the nucleon. Obviously, this should lead to an increase in the strong coupling between the partons. We regard this fact as a strict indication for establishing the mode of their correlated behavior.

In this mode, the nucleons interact in collisions not through slow (uncorrelated) partons, but as integral objects. Moreover, the slow partons, which are non-relativistic, are not formed at all in this mode. (This should not lead to significant distortion of asymptotic estimate (8), because only a limited number of partons are cut off.) The reason is actually indicated above. Namely, the appearance of slow partons leads to an increase in the length of the nucleon and, hence, to a decrease in the density of the partons. The latter property leads to an increase in the coupling between the partons, and this prevents the appearance of non-relativistic slow partons which inevitably lag behind the bulk of other (relativistic) 
partons during the longitudinal motion. Practically, this means stopping the growth of the longitudinal size of nucleons.

The simplest model that parametrically describes the evolution of the longitudinal size of nucleons looks as follows. We use a purely geometric interpretation, i.e. we assume that the nucleon at rest can be represented as a regular ball with a three-dimensional radius $R_{\mathrm{v}}^{2}=(3 / 2) \mathcal{R}_{\mathrm{v}}^{2}$. In this case the linear growth of the longitudinal radius $R_{L}$ of the nucleon is written as

$$
R_{L}=R_{\mathrm{v}}\left[1+\xi\left(\frac{E}{M}-1\right)\right] .
$$

Here $E=\sqrt{s} / 2$ is the energy of the nucleon, $M$ is its mass, and $\xi$ is a parameter. We estimate $\xi$ from the condition that three-dimensional density of the partons reaches a critical (small) value $\rho_{c}$. Namely, assuming the spatial volume of the fast nucleon is $2 R_{L} \pi \mathcal{R}^{2}$, we have $\rho(s)=\varrho /\left(2 R_{L}\right)$, where the two-dimensional density $\varrho$ is given in (3). The critical density $\rho_{c}$ can be estimated as the inverse volume $V_{c}=(4 / 3) \pi r_{c}^{3}$, where $r_{c}$ ("confinement radius") determines the maximum distance by which the partons can move away from each other. The condition $\rho\left(s_{1}\right)=\rho_{c}$ gives

$$
2 R_{\left.L\right|_{E=E_{1}}}=V_{c} \varrho,
$$

hence we get $\xi$. The result is very sensitive to $r_{c}$. In particular, assuming $r_{c}=1 \mathrm{fm}$ (should be compared with $R_{\mathrm{v}}=0.74 \mathrm{fm}$ ), we get $\xi=0.0060$ at $\sqrt{s_{1}}=2 \mathrm{TeV}$ and $\xi=0.0017$ at $\sqrt{s_{1}}=7 \mathrm{TeV}$. Assuming $r_{c}=1.2 \mathrm{fm}$, we get $\xi=0.0110$ and $\xi=0.0032$, respectively.

Table 3 shows the results for $R_{L}$ in the case $r_{c}=1 \mathrm{fm}$ at $\sqrt{s_{1}}=2 \mathrm{TeV}$ and $\sqrt{s_{1}}=7 \mathrm{TeV}$. Recall that for $s>s_{1}$ the growth of $R_{L}$ stops, and the three-dimensional local density of the partons is constant.

Table 3. The longitudinal radius $R_{L}$ of the nucleon.

\begin{tabular}{llllll}
\hline$\sqrt{\mathbf{s}}[\mathrm{GeV}]$ & $\mathbf{1 0}$ & $\mathbf{5 0}$ & $\mathbf{5 0 0}$ & $\mathbf{2 0 0 0}$ & $\mathbf{7 0 0 0}$ \\
\hline$R_{L}[\mathrm{fm}]\left(\sqrt{s_{1}}=2 \mathrm{TeV}\right)$ & 0.76 & 0.85 & 1.92 & 5.47 & 5.47 \\
$R_{L}[\mathrm{fm}]\left(\sqrt{s_{1}}=7 \mathrm{TeV}\right)$ & 0.75 & 0.77 & 1.08 & 2.77 & 5.47 \\
\hline
\end{tabular}

\section{Discussion}

We have investigated the shape of free moving fast nucleons (in the limit $t=0$ in elastic collisions, excluding electromagnetic interactions). The analysis has been carried out in the extended parton model, which consistently takes into account the transverse motions of the partons. The choice of the parton model as a research tool is caused by the impossibility of solving the problem by means of perturbative QCD. At the same time, the extended parton model makes it possible to understand the behavior of partons from the point of view of field theory without a detailed description of the dynamics of their interactions. The idea of expanding the parton model by means of taking into account the transverse motions was formulated in [6] and then developed in [12]. In the given work to solve the aforementioned problem, we use additional ideas external to the parton model.

The general idea is based on the assumption that the sizes of nucleons are comparable to the sizes of their interaction region. Physically, this is caused by the short-range property of strong interactions. In the case of transverse directions, this is strongly supported by the observation that the mean-square transverse radius $\mathcal{R}^{2}$ of the nucleons, predicted in the parton model, grows with the energy in the same way as the transverse mean-square distance $\left\langle b^{2}\right\rangle$ between the centers of their collision. This allows us to postulate equality $\mathcal{R}^{2}=\left\langle b^{2}\right\rangle / 2$ which is to be satisfied for any $s \geq s_{0}$, where $s_{0}$ is the energy above which the colliding nucleons cease to overlap with each other in the impact parameter plane $\left(\sqrt{s_{0}}\right.$ is estimated as $10 \mathrm{GeV}$ [11]). Similarly, the longitudinal sizes of the nucleons should correlate with the longitudinal sizes of their interaction region. In particular, if the longitudinal size of the interaction region grows linearly with the energy, then the longitudinal sizes of the nucleons must also grow linearly, and vice versa. 
Another additional point is the assumption that the coupling between the partons increases as their spatial density decreases. We interpret this increase as a reason for the transition to the mode of correlated motion of the partons at the energy above a certain $s_{1}\left(s_{1}>s_{0}\right)$. In this mode the transverse sizes of the nucleon grow faster than in the uncorrelated mode, but the RMS transverse momenta of the partons become limited. Concurrently, a hollow is formed inside the nucleon with a radius growing in proportion to its outer transverse radius. The hollow prevents a decrease in the local density of partons with the accelerated growth of the outer radius. The longitudinal size of the nucleon cannot grow in this mode, since the correlated behavior of the partons does not imply the presence of slow (non-relativistic) partons.

The quantitative estimates made in this article are mainly illustrative. However, they give an idea of the scale of the parameters and the trend of their change with the energy. For more accurate estimates a more detailed dynamic model is needed. Unfortunately, it was not possible to evaluate the basic parameter $s_{1}$ of the transition from the one mode to another, but this is a common difficulty in determining the transition point from $\ln s$ to $\ln ^{2} s$.

An important conclusion that can be drawn from the above results is that the longitudinal sizes of the nucleons have a decisive influence on their observable properties (despite the fact that the longitudinal sizes themselves are not directly observable). The reason lies in the fundamental dependence of the spatial density of partons on the longitudinal size of the nucleon. When the density drops to a critical value, the mode of motion of the partons changes from uncorrelated to correlated. The observed manifestations of this transition are the changes in the behavior of the slope of the diffraction cone and of the total cross section. The increase in the coupling between the partons accompanying the transition can also serve as a qualitative explanation for the observed [14] increase with the energy in the ratio of the elastic to inelastic cross section. The latter issue will be discussed elsewhere.

On the whole, the above results provide a better understanding of the processes occurring in the soft elastic collisions of nucleons at high energies, and can serve as a guide for further studies.

Funding: This research received no external funding.

Institutional Review Board Statement: Not applicable.

Informed Consent Statement: Not applicable.

Data Availability Statement: Not applicable.

Acknowledgments: The author is grateful to R.A. Ryutin for useful discussions.

Conflicts of Interest: The authors declare no conflict of interest.

\section{References}

1. Gribov, V.N. Strong Interactions of Hadrons at High Energies; Ericson, T., Landshoff, P.V., Eds.; Cambridge University Press: New York, NY, USA, 2009.

2. Feinberg, E.L. Inelastic diffraction processes at high energies. Usp. Fiz. Nauk 1956, LVIII, 193. [CrossRef]

3. Gribov, V.N.; Ioffe B.L.; Pomeranchuk, I.Y. At what distances do high energy interactions occur? Yad. Fiz. 1965, 2, 768.

4. Low F.E.; Gottfried, K. Classical space-time concepts in high-energy collisions. Phys. Rev. D 1978, 17, 2487. [CrossRef]

5. Schrempp B.; Schrempp, F. Strong interactions-A tunnelling phenomenon? Theory and application to hadronic diffraction. Nucl. Phys. B 1980, 163, 397. [CrossRef]

6. Gribov, V.N. Space-time description of the hadron interaction at high energies. Lecture given at the VIII Winter Petersburg NPI School. 1973. arXiv 2000, arXiv:hep-ph/0006158.

7. Petrov, V.A.; Ryutin, R.A. High-energy scattering versus static QCD strings. Mod. Phys. Lett. A 2015, 30, 1550081. [CrossRef]

8. Nekrasov, M.L. Charge-exchange reactions from the standpoint of the parton model. Eur. Phys. J. A 2015, 51, 148. [CrossRef]

9. Nekrasov, M.L. Non-coherent contributions in charge-exchange reactions and $\eta-\eta^{\prime}$ mixing. Eur. Phys. J. A 2017, 53, 94. [CrossRef]

10. Blok, B.; Frankfurt, L.; Strikman, M. On the shape of a rapid hadron in QCD. Phys. Lett. B 2009, 679, 122. [CrossRef]

11. Petrov, V.A.; Okorokov, V.A. The size seems to matter or where lies the "asymptopia"? Int. J. Mod. Phys. A 2018, 33, 1850077. [CrossRef]

12. Nekrasov, M.L. On the transverse size of hadrons at asymptotically high energies. Mod. Phys. Lett. A 2020, 35, 2050314. [CrossRef] 
13. Amaldi, U. Small-Angle Physics at the Intersecting Storage Rings Forty Years Later; CERN Yellow Report CERN-2012-004; CERN: Geneva, Switzerland, 2012.

14. Antchev, G. First measurement of elastic, inelastic and total cross-section at $\sqrt{s}=13 \mathrm{TeV}$ by TOTEM and overview of cross-section data at LHC energies. Eur. Phys. J. C 2019, 79, 103. [CrossRef]

15. Schegelsky V.A.; Ryskin, M.G. Diffraction cone shrinkage speed up with the collision energy. Phys. Rev. D 2012, 85, 094024. [CrossRef]

16. Donnachie, A.;Landshoff, P.V. Small $t$ elastic scattering and the $\rho$ parameter. Phys. Lett. B 2019, 798, 135008. [CrossRef]

17. Akesson, T. Multiplicity Distributions in $p-\alpha$ and $\alpha-\alpha$ Collisions in the CERN ISR. Phys. Lett. B 1982, 119, 464. [CrossRef]

18. Arnison, G. Transverse Momentum Spectra for Charged Particles at the CERN Proton anti-Proton Collider. Phys. Lett. B 1982, 118, 167. [CrossRef]

19. Khachatryan, V. (CMS Collaboration). Transverse-momentum and pseudorapidity distributions of charged hadrons in $p p$ collisions at $\sqrt{s}=0.9$ and 2.36 TeV. J. High Energy Phys. 2010, 2, 041. [CrossRef]

20. Khachatryan, V. (CMS Collaboration). Transverse-Momentum and Pseudorapidity Distributions of Charged Hadrons in $p p$ Collisions at $\sqrt{s}=7$ TeV. Phys. Rev. Lett. 2010, 105, 022002. [CrossRef] [PubMed]

21. Troshin, S.M.; Tyurin, N.E. Reflective scattering from unitarity saturation. Int. J. Mod. Phys. A 2007, 22, 4437. [CrossRef]

22. Troshin, S.M.; Tyurin, N.E. A note on the relations between elastic and inelastic interactions and increasing ratio $\sigma_{e l}(s) / \sigma_{t o t}(s)$ at the LHC. Mod. Phys. Lett. A 2019, 34, 1950259. [CrossRef]

23. Dremin, I.M. Interaction region of high energy protons. Phys. Usp. 2015, 58 61. [CrossRef]

24. Dremin, I.M. The multilayer structure of protons. Eur. Phys. J. C 2020, 80, 172. [CrossRef] 